\title{
Sistemas de calidad y desempeño empresarial: estudio de caso en empresas cárnicas en una región del noroeste de México
}

\author{
Michaelene Huerta Dueñas*, Sergio A. Sandoval Godoy*, \\ Juan Martín Preciado Rodríguez* \\ Centro de Investigación en Alimentación y Desarrollo A. C. (CIAD). \\ Hermosillo, Sonora, México. \\ Recibido: 16 de agosto del 2016 / Aprobado: 28 de setiembre del 2016
}

RESUMEN: En este artículo se realiza un análisis exploratorio de la medición del uso de los sistemas de calidad y sus implicancias en el desempeño empresarial, tomando como referencia un estudio de caso en cuatro empresas cárnicas ubicadas en una ciudad en el noroeste de México. La contribución principal del trabajo se basa en un diseño metodológico mixto, analizado mediante la ponderación preferencial de los criterios. Los resultados tejen coincidencias con otras investigaciones, concluyendo que los sistemas de calidad incrementan el desempeño empresarial en los montos de producción y ventas.

Palabras clave: sistemas de calidad / normatividades de calidad / metodologías de calidad / desempeño empresarial

\section{Quality systems and business performance: case study in meat producing companies in a region of northwestern México}

AвSTRACT: In this article an exploratory analysis of the measurement of use of quality systems and their implications in business performance is done, taking as reference a case study in four meat producing companies of a Northern Mexican County. The main contribution of this work is based on a mixed methodology analyzed by means of the Preferential Weighting of Criteria. The results thread coincidences with other investigations, that conclude that quality systems increase enterprise performance in the productions and sales amounts.

Keywords: quality systems / quality normativities / quality methodologies / enterprise performance

Correos electrónicos: michaelene@estudiantes.ciad.mx, ssandoval@ciad.mx y mpreciado@ciad.mx 


\section{INTRODUCCIÓN}

La necesidad de garantizar la mejora continua de los productos a lo largo de toda la cadena productiva ha provocado un interés creciente de las gerencias por la aplicación de los sistemas de calidad (SC). Estos se definen como el conjunto de normatividades y metodologías que se interrelacionan entre sí para dirigir y controlar los procesos productivos de las empresas, a partir de un enfoque en el que prevalece una cultura y orientación hacia la calidad y la mejora continua (Camisón, BoronatNavarro, Villar-López y Puig-Denia, 2009; Álvarez-García, Vila-Alonso, Fraiz-Brea y Río-Rama, 2014). Las normatividades de calidad (NC) son el conjunto de certificaciones y acreditaciones que contienen las especificaciones técnicas y criterios precisos para los procedimientos de gestión exigidos por un organismo regulatorio, los cuales otorgan la garantía de que un producto está en conformidad con ciertas normas (Organización de las Naciones Unidas para la Alimentación y la Agricultura [FAO], 2004a). Adicionalmente, las metodologías de calidad (MC) son el cúmulo de técnicas y herramientas ${ }^{1}$ que constituyen la dimensión operativa necesaria para apoyar el aseguramiento de la calidad y la puesta en marcha de los procesos de mejora continua en la empresa (ÁlvarezGarcía et al., 2014).

En los últimos años, hay un creciente interés por los impactos que generan los sistemas de calidad en el desempeño empresarial. Este se entiende como la capacidad de las empresas para obtener el desempeño operativo y financiero esperado (Calantone, Cavusgil y Zhao, 2002), derivado de al menos cuatro indicadores: eficiencia en el uso de la tecnología, montos de producción, ventas y exportaciones. De hecho, se ha comprobado que tanto las normatividades como las metodologías conllevan el incremento del desempeño operacional; no obstante, algunas investigaciones ponen en entredicho la existencia de una relación positiva entre las certificaciones y el desempeño financiero (Lo, Yeung y Edwin-Cheng, 2011; Moura-Duarte, Ledur-Brito, Di-Serio y Silveira-Martins, 2011; Chikuku, Chinguwa y Macheka, M., 2012; Tarí-Guilló y Pereira-Moliner, 2012; Ul- Hassan, Mukhtar, Ullah-Qureshi y Sharif, 2012; Marín-Vinuesa, 2013; Morelos-Gómez,

1 Las técnicas de calidad son el conjunto de procedimientos necesarios para alcanzar un objetivo, tales como seis sigma y benchmarking, mientras que las herramientas de calidad son los instrumentos que se utilizan para llevar a cabo una actividad, como el control estadístico del proceso y los diagramas de flujo. 
Fontalvo y Vergara, 2013; Mahnaz, 2014; Pérez-Méndez y MachadoCabezas, 2015).

En el caso de las empresas cárnicas de México, las certificaciones y metodologías de calidad, aun cuando su empleo es todavía limitado y restringido para unas cuantas (López-Palacios, Muñoz-Rodríguez, Leos-Rodríguez y Cervantes-Escoto, 2010; Álvarez-García et al., 2014), ha generado resultados positivos en términos del crecimiento de la producción y exportación. Además, han sido identificadas como ineludibles para incrementar la rentabilidad de las empresas (Boyer, y Freyssenet, 2003).

Cabe señalar que, en la revisión de la literatura, se detecta que los estudios sobre los efectos de los sistemas de calidad se han realizado mayormente en la industria automotriz, metal-mecánica, electrónica, del mueble, bebida y de servicios, con base en las normas ISO (establecidas por la Organización Internacional para la Estandarización) y el modelo europeo de excelencia, al encontrarse una ausencia clara de otros estándares que aparentemente podrían ser de uso común en las agroindustrias alimentarias. Dentro de la industria alimentaria se reporta el uso de algunas certificaciones de calidad, mas no se analiza el efecto que estas tienen sobre el desempeño empresarial. También se observa un vacío de información documentada dentro del manejo integrado de los sistemas de calidad, tanto de las metodologías como de las certificaciones.

Con el propósito de cubrir parte de estas ausencias y con la finalidad de identificar cómo es que los sistemas de calidad en las empresas cárnicas bovinas y porcinas -dedicadas a comercializar productos en el mercado nacional y de exportación- inciden significativamente en el desempeño empresarial, se presenta un estudio de caso. Tiene como referencia a cuatro empresas de la ciudad de Hermosillo, capital del estado de Sonora, ubicada en el noroeste de México, con fronteras que colindan por el norte con el estado de Arizona, Estados Unidos; con el Golfo de California en el litoral oeste; al este con Chihuahua y al sur con Sinaloa.

El trabajo está estructurado en cuatro partes: primero, se realiza una revisión de la literatura, para dar cuenta de las particularidades que adquieren la aplicación de los sistemas de calidad en la industria manufacturera. Así, se infiere que el desempeño empresarial no depende tanto del tamaño de la firma, sino del conocimiento especializado 
que se tiene de dichos sistemas, como del giro empresarial y los requerimientos de inversión de capital. Seguidamente se presenta la metodología de investigación, donde se describen las características del trabajo, las empresas seleccionadas, el tamaño de la población, los instrumentos utilizados y el método de análisis. En el tercer apartado se presentan y discuten los resultados de la investigación y, finalmente, se exponen las conclusiones más relevantes.

\section{REVISIÓN DE LA LITERATURA}

Aunque la calidad no es un tema nuevo, al menos en las últimas cuatro décadas del desarrollo de la sociedad industrial moderna han resurgido nuevos parámetros para su medición y numerosos argumentos para su estudio. En algunos segmentos de la industria se ha identificado que la calidad constituye una de las seis estrategias que permiten el incremento de los niveles de rentabilidad en las empresas. Se destaca que la calidad, por ejemplo en productos de gama alta ${ }^{2}$, desempeña un papel mayor que las estrategias de diversidad de la oferta, flexibilidad productiva, volumen de producción, innovación y reducción permanente de costos (Boyer y Freyssenet, 2003).

Lo anterior ha generado que durante los últimos años se hayan realizado diversos estudios acerca de las implicancias que conlleva la implementación de sistemas de la calidad. No obstante, estas se han centrado únicamente en el análisis de certificaciones ISO 9000Q ${ }^{3}$ y en el modelo europeo de excelencia dentro de ciertas industrias del sector manufacturero y de servicios, principalmente en España, Estados Unidos, China y otros países de Europa.

Numerosas investigaciones que analizan el impacto que ejercen las normatividades sobre el desempeño operacional concluyen que es positivo (Ul-Hassan et al., 2012; Morelos-Gómez et al., 2013; Sánchez-Ollero, García-Pozo y Marchante-Mera, 2014). Ahora bien, la relación entre los estándares de calidad con el desempeño financiero es controversial,

2 Los productos de gama alta se refieren a todos aquellos artículos de lujo con alto valor comercial y calidad superior, dirigidos a los segmentos de población de clase alta.

3 Normatividad que especifica el nivel de calidad esperado en el servicio al cliente en empresas de servicio. 
puesto que algunos trabajos argumentan que las empresas certificadas obtienen un mejor desempeño que las que carecen de normatividades de calidad (Tarí-Guilló y Pereira-Moliner, 2012; Ul-Hassan et al., 2012; Morelos-Gómez, et al., 2013; Mahnaz, 2014; Pérez-Méndez y MachadoCabezas, 2015), mientras que otros no encuentran evidencia alguna de dicha relación (Heras y Landín, 2011; Lo et al., 2011; Moura-Duarte et al., 2011; Chikuku et al., 2012; Marín-Vinuesa, 2013).

Diversos autores sugieren que esta disputa es ocasionada por las distintas razones que guían a las empresas a certificarse, ya que, en efecto, las empresas que se certifican por motivos internos, relacionados con la mejora de la eficiencia y el control de la empresa, consiguen un mejor desempeño financiero (Llopis y Tarí-Guilló, 2003; MartínezCosta y Martínez-Lorente, 2008), respecto a aquellas que lo hacen presionadas por motivos externos, referidos a la imagen que proyecta la calidad y a las presiones por parte de los gobiernos y los consumidores (Heras, Landín y Casadesús, 2006; Marín-Vinuesa, 2013). Cabe aclarar, como sugieren Tarí-Guilló y Pereira-Moliner, (2012) que el tamaño de las empresas no es un factor clave para entender la relación entre las certificaciones y el desempeño financiero, por lo que ser una empresa pequeña no es una dificultad en la adopción de normatividades de calidad. Sin embargo, Heras y Landín (2011) sostienen que las empresas con mejor rentabilidad tienden con mayor frecuencia a certificarse.

Por otra parte, existe una carencia de investigaciones que vinculan la relación entre las normatividades y las metodologías de calidad. Sin embargo, se reporta que las empresas que emplean una mayor cantidad de metodologías de calidad obtienen un mejor desempeño operacional, sin importar el tamaño de la empresa (Ahmed y Hassan, 2003). Álvarez-García et al. (2014) afirman que no existen diferencias significativas en la utilización de metodologías de calidad en las empresas pequeñas y medianas. No obstante, las metodologías de calidad cuantitativas como el control estadístico del proceso, seis sigma, Análisis de Modo y Efecto de Fallas, entre otras, son utilizadas con menor frecuencia que las de tipo cualitativo, puesto que requieren un mayor grado de conocimiento especializado e implican una mayor inversión monetaria, ya que son estas las que se relacionan en mayor grado con el desempeño operacional de las empresas (Heras, Marimon y Casadesús, 2009). 
De hecho, Heras, et al., (2009) comprobaron que las empresas con sistemas de calidad avanzados emplean metodologías de calidad cuantitativas y muestran más interés en su uso. Además, Álvarez-García, et al., (2014) reportan que sobre todo en empresas de servicios las metodologías de calidad cuantitativas como el diagrama de Pareto, el diagrama de correlación y la casa de la calidad, no se implementan debido a que se desconocen; en caso contrario, las metodologías cualitativas son más conocidas y no requieren de infraestructura, ni excesiva inversión inicial. Entre estas últimas, las más utilizadas son las encuestas de satisfacción, los grupos de mejora y los sistemas de quejas y sugerencias. Por lo anterior, concluyen que de acuerdo a la situación particular de cada empresa, se seleccionará el tipo de metodología a emplear.

De acuerdo con la revisión de la literatura efectuada, todo parece indicar que la instrumentación de sistemas de calidad influye de manera positiva en el desempeño de las empresas, independientemente de su tamaño, pero dependiendo del conocimiento especializado que se tiene de estos aspectos, además de su giro productivo y requerimientos de inversión de capital. Lo anterior aplica a las empresas cárnicas ubicadas en Hermosillo (Sonora, México), registradas con la certificación de Tipo Inspección Federal (TIF) ${ }^{4}$, como se verá enseguida.

\section{METODOLOGÍA}

El procedimiento que siguió la investigación fue de corte exploratorio con un método mixto. Específicamente se inicia con la identificación de los aspectos claves para la medición de los sistemas de calidad y el desempeño empresarial, así como la recopilación de información documental necesaria para complementar la información teórica relacionada con cada una de las variables analizadas. Seguidamente, se contactó a las empresas que participarían en el trabajo, con la finalidad de asegurar el acceso a la información. Subsiguientemente, se diseñaron, validaron y aplicaron encuestas y entrevistas a los sujetos informantes, para analizarlas y obtener las conclusiones de este estudio.

4 Reconocimiento que la Secretaría de Agricultura, Ganadería, Desarrollo Rural, Pesca y Alimentación (Sagarpa) otorga a las empresas que producen, procesan, almacenan y distribuyen alimentos cárnicos y sus derivados. Este sello garantiza que el producto cumple con todas las normas y exigencias del gobierno mexicano en cuanto al manejo de sanidad e inocuidad. 


\subsection{Población del estudio}

El trabajo está centrado en empresas cárnicas, debido a la importancia económica que genera la industria de la carne en México, que sobresale a nivel mundial dentro de los diez principales productores y exportadores de carne porcina, además de un crecimiento importante en la producción de carne bovina (Departamento de Agricultura de los Estados Unidos [USDA], 2015). Del mismo modo, la carencia de estudios que evalúen la relación entre los sistemas de calidad y el desempeño empresarial en el sector agropecuario es otro elemento que influye en la selección de la población.

Dentro de las empresas cárnicas, se seleccionaron empresas ubicadas en Hermosillo, Sonora, apegadas a la certificación TIF con base en el listado creado por el Servicio Nacional de Sanidad, Inocuidad y Calidad Agroalimentaria. En el municipio de Hermosillo hay un total de seis empresas dedicadas a diversas fases del proceso de producción de la carne. Cabe mencionar que ellas aportan el 18,9 \% de la producción total nacional de carne de cerdo, situando a Sonora como el segundo estado con mayor producción en México, solo antecedido por Jalisco, con 19,1 \% (Fideicomisos Instituidos en Relación con la Agricultura [FIRA], 2014). De igual manera, colocan a Sonora como el primer exportador nacional de puerco con 62,5 \% (FIRA, 2014; Sagarpa, 2014). De las seis empresas en dicha localidad, únicamente cuatro accedieron a colaborar en este estudio, por lo que la selección fue no probabilística e intencional.

Por cuestiones de confidencialidad de las empresas seleccionadas, en apartados subsiguientes serán denominadas por las literales del alfabeto A, B, C y D. Cabe señalar que si bien el análisis realizado es de corte exploratorio y con una reducida población, esta investigación es suficiente para marcar una tendencia en cuanto al impacto de los sistemas de calidad sobre el desempeño empresarial en la industria de la carne.

\subsubsection{Características generales de la población}

Para un mejor análisis e interpretación de los resultados derivados de este estudio, fue necesario examinar algunas características de las 
empresas, descritas posteriormente como: antigüedad, ventas anuales, tipo de mercado ${ }^{5}$, cadena de suministro ${ }^{6}$, entre otras.

La empresa A se dedica al sacrificio de ganado vacuno y al deshuese de canales o carcasas ${ }^{7}$ de cerdo de diversas empresas y ganaderos de la región. Es una empresa manufacturera de tamaño pequeño ${ }^{8}$, fundada en el año 2006, que emplea al menos 120 trabajadores y genera 24 millones de pesos anuales en promedio.

Por su parte, la B es una empresa grande, creada en 1991, con 162 empleados y 564 millones de pesos en ventas por año. Las fases que integran sus procesos productivos son: la crianza y engorde de reses, el procesamiento, empaque y distribución de carne bovina para el mercado local, nacional y asiático.

La $\mathrm{C}$ es una empresa pequeña con más de 23 años de trayectoria. Sus montos anuales de ventas ascienden a 22 millones de pesos con menos de 19 empleados. Se dedica a la producción y comercialización de carne de ave, res y puerco destinada a abastecer la demanda de diversas cocinas industriales y empresas de servicios en distintas localidades de México. De las empresas estudiadas, esta es la única que cuenta con otra planta fuera del estado de Sonora; además, recientemente fue adquirida por una firma trasnacional.

5 Definir el mercado significa delinear sus límites, de tal manera que este contenga todos los bienes sustitutos y excluya aquellos que no lo son (Shepherd, 1999).

6 La cadena de suministro o de valor es la integración e interrelación de diversos procesos claves del negocio, desde las materias primas hasta el producto terminado (Chavez, J. y Torres-Rabello, R., 2012). En la industria de la carne, la cadena de valor integra el engorde y crianza del animal (producción primaria), sacrificio del ganado, industrialización o procesamiento y distribución del producto.

7 Canal es el cuerpo del animal después del sacrificio, sin vísceras, riñones, cabeza, cola, genitales, ubres, desangrados y con extremidades cortadas a nivel articulación carpo-metacarpiana y tarso-metatarsiana.

8 El tamaño de las empresas se estableció de acuerdo a la estratificación del Diario Oficial de la Federación a partir de la fórmula: puntaje de la empresa = (número de empleados) $(10 \%)+($ monto de ventas) $(90 \%)$. Dentro del sector industrial, las empresas pequeñas deben tener un puntaje menor o igual a 95 ; las de tamaño mediano, una puntuación inferior o igual a 250, y las empresas grandes, un valor por encima o igual a 251. 
Finalmente, la $\mathrm{D}$ tiene la característica de ser una empresa de mucha tradición en la región, con más de 30 años de operación. Es una empresa grande que genera 330 empleos en promedio. Su volumen de ventas anuales es de 1020 millones de pesos. Las fases que integran sus procesos productivos son: la producción primaria, la industrialización y la distribución de carne bovina, porcina y avícola. Sus productos se dirigen principalmente al mercado regional y nacional, y exporta a Estados Unidos y Asia los remanentes sobrantes del mercado mexicano.

\subsection{Instrumentos para el acopio de información}

La recolección de información del estudio se llevó a cabo en dos etapas. La primera consistió en diseñar dos encuesta estructuradas, al tomar como referencia los principios productivos de Boyer y Freyssenet, M. (2003), e indicadores de calidad extraídos de Heras, Bernardo y Casadesús (2007) y Camisón et al. (2009). Los instrumentos recababan algunas características generales de las empresas analizadas, e información referente a las normatividades y metodologías de calidad. Cada encuesta se diseñó sobre la base de los sujetos informantes. Por el tipo de información contenida, uno fue aplicado a los gerentes de calidad y producción, y el otro a los directivos de compras y ventas, debido a que dichos puestos son claves para obtener datos más cercanos a los analizados en esta investigación.

Las preguntas de ambas encuestas eran abiertas y cerradas -aunque en su mayoría eran preguntas de este último tipo-, medidas a través de la escala de Likert con valores de 1 (nunca), 2 (muy pocas veces), 3 (algunas veces), 4 (casi siempre) y 5 (siempre). La validez de los instrumentos se obtuvo tras la revisión de cinco expertos en cada tipo de encuesta, quienes evaluaron la pertinencia de las preguntas, la escala de medición, el lenguaje, entre otros aspectos. En las cuatro empresas se aplicaron un total de dieciséis encuestas, donde ocho fueron a gerentes de calidad y producción, y las restantes a compras y ventas.

Con la finalidad de medir el desempeño empresarial y obtener datos más precisos, fue necesaria una segunda etapa de investigación. Esta consistió en realizar un cuestionario guía de entrevista dirigido a gerentes de compras y ventas. Del mismo modo que en las encuestas, la entrevista se validó con cinco expertos y, posteriormente, se entrevistó un total de ocho gerentes en las empresas estudiadas. 


\subsection{Método de análisis}

Puesto que la mayoría de la información recabada en los distintos instrumentos fue de tipo cualitativo, se requirió transformar los datos a cuantitativos mediante la ponderación preferencial de criterios propuesta por Romero (1996). Esta es utilizada para el análisis de decisiones multicriterio, donde se clasifican los criterios por orden de importancia, y se le asigna el número 1 al criterio más importante, 2 al siguiente en importancia, y sucesivamente hasta asignar el número $\mathrm{n}$ al criterio menos importante. De este modo, para establecer los pesos compatibles se aplicó la ecuación 1, donde $r_{j}$ es el lugar o posición que ocupa el criterio j-ésimo en la clasificación establecida.

$$
W_{j}=\frac{\frac{1}{r_{j}}}{\sum_{i=1}^{n} \frac{1}{r_{j}}}
$$

Para determinar el indicador de las normatividades y metodologías de calidad, se emplearon las fórmulas 2 y 3 , según la variable analizada. El peso SC es el valor de importancia que tienen las normatividades y las metodologías de calidad dentro de los sistemas de calidad. Puesto que las metodologías se relacionan con la mejora continua y constituyen la dimensión operativa necesaria para apoyar la implementación y supervisión de las normas de calidad, a las primeras se les asignó un peso de 0,60, mientras que a las normas, 0,40. El peso $\mathrm{MC}$ representa el valor que tienen tanto las técnicas como las herramientas dentro de las metodologías de calidad. Debido a que las herramientas integran a las técnicas de calidad, se les dio un valor de 0,30, y a las técnicas, 0,70.

$$
\begin{aligned}
& \mathrm{NC}=\left(\mathrm{W}_{\mathrm{j}}\right)(\text { peso } \mathrm{SC}) \\
& \mathrm{MC}=\left(\mathrm{W}_{\mathrm{j}}\right)(\text { peso } \mathrm{MC})(\text { peso } \mathrm{SC})
\end{aligned}
$$

La ponderación preferencial de los criterios permitió cuantificar el grado de utilización de diversas normatividades y metodologías de calidad, y a partir de dichos valores, estimar de manera conjunta el nivel de uso de los sistemas de calidad en cada una de las empresas analizadas. Una vez obtenidas las ponderaciones, fue posible contrastar y analizar los valores de los sistemas de calidad con los del desempeño empresarial, y comprobar la hipótesis antes planteada. 


\section{RESULTADOS Y DISCUSIÓN}

Como se mencionó anteriormente, para cuantificar los sistemas de calidad se utilizó la ponderación preferencial de criterios. En primer lugar se obtuvieron los índices de peso correspondientes a las normatividades de calidad (tabla 1). Esta variable tiene dos niveles: sí y no, es decir, la empresa está certificada o no en determinada normatividad. Una vez determinados los valores de estimación de cada uno de los niveles mediante las ecuaciones 1 y 2 , se obtuvieron los indicadores que aparecen en la tabla 2.

Tabla 1

Índices de pesos preferenciales de las normatividades de calidad

\begin{tabular}{|c|c|c|c|c|}
\hline Variable & Niveles & Estimación & Peso SC & Indicador \\
\hline (1) & Sí & $\frac{\frac{1}{1}}{\frac{1}{1}+\frac{1}{2}}=0,66$ & & 0,27 \\
\hline 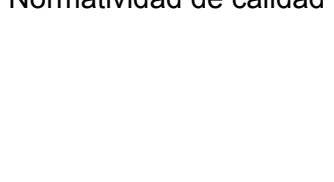 & No & $\frac{\frac{1}{2}}{\frac{1}{1}+\frac{1}{2}}=0,33$ & 0,40 & 0,13 \\
\hline
\end{tabular}

Elaboración propia con base en la información recopilada en las encuestas y entrevistas

La certificación más implementada es HACCP (Análisis de riesgos y de puntos críticos de control), utilizada con la finalidad de complementar a los estándares de calidad previamente implementados. Esta es seguida por las normas establecidas en el Codex Alimentarius USDA y por el Servicio Nacional de Investigación Veterinaria y Cuarentena (NVRQS), las cuales son exigidas por el mercado estadounidense y asiático. Por último, las menos utilizadas son MCS (México Calidad Suprema), un sello implementado voluntariamente por parte de las empresas para ofrecer a los consumidores una garantía más de calidad en sus productos; la norma MAFF (Ministerio de Agricultura, Silvicultura e Industria Pesquera), establecida por el mercado asiático, y las acreditaciones gestionadas mediante Sagarpa, que esta dependencia emite para garantizar a determinado país que la empresa cumple con los requerimientos que solicita. 
Tabla 2

Estimación de los índices de pesos preferenciales de las normatividades de calidad por empresa

\begin{tabular}{ccccccc}
\hline \multirow{2}{*}{$\begin{array}{c}\text { Normatividad } \\
\text { de calidad }\end{array}$} & Producto & \multicolumn{6}{c}{ Empresa } & \multirow{2}{*}{ Total } \\
\cline { 3 - 6 } & & $\mathbf{A}$ & $\mathbf{B}$ & $\mathbf{C}$ & $\mathbf{D}$ & \\
\hline \multirow{2}{*}{ HACCP } & Puerco & 0,27 & 0,13 & 0,13 & 0,27 & $\mathbf{0 , 2 2}$ \\
& Res & 0,27 & 0,27 & 0,13 & 0,27 & \\
Codex Alimentarius & Puerco & 0,27 & 0,13 & 0,13 & 0,13 & $\mathbf{0 , 2 0}$ \\
& Res & 0,27 & 0,27 & 0,13 & 0,27 & \\
USDA & Res & 0,27 & 0,13 & 0,13 & 0,27 & $\mathbf{0 , 2 0}$ \\
NVRQS & Res & 0,27 & 0,13 & 0,13 & 0,27 & $\mathbf{0 , 2 0}$ \\
\cline { 5 - 6 } MCS & Puerco & 0,13 & 0,13 & 0,13 & 0,27 & $\mathbf{0 , 1 7}$ \\
& Res & 0,13 & 0,13 & 0,13 & 0,27 & \\
MAFF & Res & 0,13 & 0,13 & 0,13 & 0,27 & $\mathbf{0 , 1 7}$ \\
Gestionada por Sagarpa & Res & 0,13 & 0,27 & 0,13 & 0,13 & 0,17 \\
& Total & $\mathbf{0 , 2 1}$ & $\mathbf{0 , 1 7}$ & $\mathbf{0 , 1 3}$ & $\mathbf{0 , 2 4}$ & $\mathbf{0 , 1 9}$ \\
\hline
\end{tabular}

Elaboración propia con base en la información recopilada en las encuestas y entrevistas

Aunado a lo anterior, las cuatro empresas analizadas presentan baja utilización de las normas de calidad, ya que solo se utilizan en un 0,19 . Este resultado concuerda con lo reportado por López-Palacios et al. (2010), quienes analizan a empresas líderes de la industria cárnica bovina en México.

La empresa con mayor número de certificaciones es la $\mathrm{D}$, seguida por las A, B y C. La A destacó como el segundo lugar, debido a que es la empresa que sacrifica el ganado bovino de diversas empresas en la localidad. Por lo tanto, debe apegarse a los estándares de calidad implementados por las empresas para las cuales sacrifica. En este tipo de empresas se debe manejar la misma certificación a lo largo de todas las fases que integran al proceso productivo de la carne, pues en caso contrario, esta se pierde.

En relación a lo ya mencionado en la metodología, A y C son empresas pequeñas, en tanto que $\mathrm{B}$ y $\mathrm{D}$ son empresas grandes, por lo cual se puede inferir que el tamaño de las empresas no determina el apego a una mayor cantidad de certificaciones, lo que concuerda con Tarí-Guilló y Pereira-Moliner (2012) para el caso de las empresas de servicios. Además, como ya se mencionó, las metodologías de calidad 
Tabla 3

Índices de pesos preferenciales de las metodologías de calidad

\begin{tabular}{|c|c|c|c|c|c|}
\hline Variable & Niveles & Estimación & Peso MC & Peso SC & Indicadores \\
\hline \multirow{4}{*}{$\begin{array}{l}\text { Técnica de } \\
\text { calidad }\end{array}$} & Alto & $\frac{\frac{1}{1}}{\frac{1}{1}+\frac{1}{2}+\frac{1}{3}+\frac{1}{4}}=0,48$ & & & 0,20 \\
\hline & Medio & $\frac{\frac{1}{2}}{\frac{1}{1}+\frac{1}{2}+\frac{1}{3}+\frac{1}{4}}=0,24$ & 0,70 & 0,60 & 0,10 \\
\hline & Bajo & $\frac{\frac{1}{3}}{\frac{1}{1}+\frac{1}{2}+\frac{1}{3}+\frac{1}{4}}=0,16$ & & & 0,07 \\
\hline & Nulo & $\frac{\frac{1}{4}}{\frac{1}{1}+\frac{1}{2}+\frac{1}{3}+\frac{1}{4}}=0,12$ & & & 0,05 \\
\hline \multirow{4}{*}{$\begin{array}{l}\text { Herramienta } \\
\text { de calidad }\end{array}$} & Alto & $\frac{\frac{1}{1}}{\frac{1}{1}+\frac{1}{2}+\frac{1}{3}+\frac{1}{4}}=0,48$ & \multirow{4}{*}{0,30} & \multirow{4}{*}{0,60} & 0,09 \\
\hline & Medio & $\frac{\frac{1}{2}}{\frac{1}{1}+\frac{1}{2}+\frac{1}{3}+\frac{1}{4}}=0,24$ & & & 0,04 \\
\hline & Bajo & $\frac{\frac{1}{3}}{\frac{1}{1}+\frac{1}{2}+\frac{1}{3}+\frac{1}{4}}=0,16$ & & & 0,03 \\
\hline & Nulo & $\frac{\frac{1}{4}}{\frac{1}{1}+\frac{1}{2}+\frac{1}{3}+\frac{1}{4}}=0,12$ & & & 0,02 \\
\hline
\end{tabular}

Elaboración propia con base en la información recopilada en las encuestas y entrevistas

se dividen en técnicas y herramientas de calidad (véase tabla 3). Los niveles de las técnicas y herramientas de calidad dependen del grado de utilización, puesto que no se implementan por igual ni con la misma 
intensidad en cada una de las empresas estudiadas. Por ello, estos se dividen en uso: alto, medio, bajo y nulo. Para estimar los indicadores correspondientes a cada uno de los niveles, se utilizaron las fórmulas 1 y 3 , como se observa en la tabla 4.

La empresa B es la que utiliza un mayor número de metodologías de calidad en sus procesos de producción, seguida por C, D y A. Como se puede observar, el aplicar un mayor número de metodologías no implica tener más certificaciones implementadas, ya que las normas no están reguladas por metodologías en específico.

Tabla 4

Estimación de los índices de pesos preferenciales de las metodologías de calidad por empresa

\begin{tabular}{|c|c|c|c|c|c|c|}
\hline \multirow{2}{*}{\multicolumn{2}{|c|}{ Metodología de calidad }} & \multicolumn{4}{|c|}{ Empresa } & \multirow{2}{*}{ Total } \\
\hline & & A & B & C & D & \\
\hline \multirow{5}{*}{ 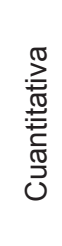 } & Seis sigma & 0,07 & 0,20 & 0,07 & 0,20 & \multirow{5}{*}{0,09} \\
\hline & Análisis de modo y efecto de fallas & 0,05 & 0,10 & 0,05 & 0,10 & \\
\hline & Auditorías internas & 0,05 & 0,20 & 0,20 & 0,05 & \\
\hline & Control estadístico del proceso & 0,03 & 0,09 & 0,09 & 0,09 & \\
\hline & Siete herramientas administrativas & 0,02 & 0,04 & 0,02 & 0,02 & \\
\hline \multirow{8}{*}{ 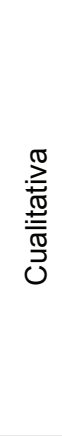 } & Benchmarking & 0,07 & 0,07 & 0,07 & 0,05 & \multirow{8}{*}{0,06} \\
\hline & Satisfacción del cliente & 0,07 & 0,10 & 0,07 & 0,05 & \\
\hline & Metodologías 5's & 0,05 & 0,05 & 0,05 & 0,07 & \\
\hline & Grupos de mejora & 0,02 & 0,04 & 0,09 & 0,02 & \\
\hline & Diagrama de flujos & 0,02 & 0,09 & 0,09 & 0,02 & \\
\hline & $\begin{array}{l}\text { Disciplina para la resolución de } \\
\text { problemas }\end{array}$ & 0,02 & 0,09 & 0,09 & 0,02 & \\
\hline & Sistema de quejas y sugerencias & 0,04 & 0,09 & 0,02 & 0,09 & \\
\hline & Lluvia de ideas & 0,02 & 0,04 & 0,09 & 0,02 & \\
\hline \multicolumn{2}{|c|}{ Total } & 0,04 & 0,09 & 0,08 & 0,06 & 0,07 \\
\hline
\end{tabular}

Elaboración propia con base en la información recopilada en las encuestas y entrevistas

Del mismo modo que las normatividades de calidad, este tipo de empresas muestra un bajo manejo de metodologías de calidad, al utilizar únicamente 0,07. Esto coincide con Álvarez-García et al. (2014), quienes encuentran el mismo resultado en empresas de servicio.

Las metodologías de calidad de tipo cuantitativo son utilizadas con mayor frecuencia y de manera más completa, incluso cuando se emplea 
un mayor número de metodologías cualitativas. Este resultado contrasta con lo reportado por Heras, I. et al. (2009), quienes encontraron que en las industrias manufactureras y de servicios, la calidad no es la principal estrategia para incrementar la rentabilidad, lo que puede ser explicado por las diferencias en el tipo de modelo utilizado y el tamaño de la muestra. Cabe aclarar que este estudio solo contempla cuatro empresas del sector agropecuario, en las que, según lo reportado por la FAO (2004b), la calidad es un componente esencial para la penetración de mercados y para incrementar la competitividad.

Una vez calculados los índices de peso de las normatividades y las metodologías de calidad, se encontró que los sistemas de calidad son utilizados en un 0,27, como se detalla en la tabla 5. La empresa D emplea en mayor grado los sistemas de calidad, después de B, A y C. Además, en dicha tabla se detallan algunos valores del desempeño empresarial (montos de producción, ventas y exportación) recabados a través de las entrevistas. La empresa D tiene mayores montos de producción y ventas, seguida por B, C y A.

Se observa que las empresas con más sistemas de calidad tienen mayores montos de producción y ventas. Sin embargo, los niveles de exportación no pueden compararse, ya que la empresa A y C no exportan debido a la carencia de clientes, y la $\mathrm{D}$ exporta únicamente los remanentes no deseados por el mercado mexicano. Así, la empresa B es la principal exportadora de carne de res, al quedar por debajo la D con carne de puerco.

Tabla 5

Comparación de los índices de pesos preferenciales con valores del desempeño empresarial

\begin{tabular}{ccccccc}
\hline Empresa & $\begin{array}{c}\text { Normatividades } \\
\text { de calidad }\end{array}$ & $\begin{array}{c}\text { Metodologías } \\
\text { de calidad }\end{array}$ & $\begin{array}{c}\text { Sistemas } \\
\text { de calidad }\end{array}$ & Producción & Ventas & Exportación \\
\hline A & 0,21 & 0,04 & $\mathbf{0 , 2 6}$ & 0 & 2 & 0 \\
B & 0,17 & 0,09 & $\mathbf{0 , 2 7}$ & 580 & 47 & 26 \\
C & 0,13 & 0,08 & $\mathbf{0 , 2 3}$ & 95 & 1,8 & 0 \\
D & 0,24 & 0,06 & $\mathbf{0 , 3 1}$ & 650 & 85 & 15 \\
\hline Total & $\mathbf{0 , 1 9}$ & $\mathbf{0 , 0 7}$ & $\mathbf{0 , 2 7}$ & & & \\
\hline
\end{tabular}

Elaboración propia con base en la información recopilada en las encuestas y entrevistas 


\section{CONCLUSIÓN}

Después de haber analizado cómo los sistemas de calidad se relacionan con el desempeño empresarial, concluimos que las empresas cárnicas con mayor propensión a utilizar sistemas de calidad obtienen mejores resultados en términos de ventas totales y producción. Sin embargo, se observa que las empresas analizadas presentan todavía una escasa instrumentación de sistemas de calidad.

Particularmente, en lo que respecta a las normatividades de calidad, este estudio se suma a otras contribuciones empíricas como las de López-Palacios et al. (2010), quienes después de analizar diez empresas líderes de carne bovina a nivel nacional, concluyen que estas cumplen mínimamente con los requisitos impuestos por las certificaciones de calidad, con el objetivo de comercializar sus productos en diversas cadenas de supermercados y en los mercados de exportación. Al igual que en esta investigación, también señalan que algunas normatividades de calidad son implementadas por presión de los clientes y con la finalidad de ganar reconocimiento y prestigio, y no para la mejora continua de los productos y procesos. Asimismo, este estudio coincide con Tarí-Guilló y Pereira-Moliner (2012) en que no se observa relación alguna entre el tamaño de la empresa y la cantidad de normatividades de calidad.

Por otra parte, en cuanto a las metodologías de calidad, los resultados encontrados confirman los hallazgos reportados por otros estudios como los de Ahmed y Hassan (2003) y Álvarez-García et al. (2014), que resaltan su reducida utilización. Incluso, se hace hincapié en que el personal directivo debe poner atención en el uso de las metodologías de calidad, pues estas permiten mejorar el desempeño operativo de las empresas.

En contraste con Heras et al. (2009), las metodologías de calidad de tipo cuantitativo se utilizan con mayor vehemencia, puesto que en este tipo de empresas la calidad juega un papel principal para captar clientes y penetrar mercados. Cabe mencionar que, a pesar de que se utilizan con mayor intensidad, se emplea un número menor en comparación a las cualitativas. Parte de este problema se debe a la falta de información y capacitación, como sugieren Dale y Mcquater (1998).

En resumen, consideramos que la presente investigación contribuye al conocimiento que favorece la conjetura de una relación positiva entre los sistemas de calidad y el desempeño de las empresas, tema que se ha estudiado durante los últimos años. De paso, se pone de manifiesto 
la necesidad que tienen las empresas de fomentar la integración de las normatividades y las metodologías como elementos constitutivos de los sistemas de calidad, aunado a que estos son una premisa indispensable en las agroindustrias de alimentos para incrementar su rentabilidad y permitir su acceso a los mercados de exportación.

Finalmente, a pesar de su carácter exploratorio, este trabajo proporciona elementos metodológicos de medición y análisis estadísticos válidos y adecuados, no solo para entender la relación entre la utilización de los sistemas de calidad con el desempeño empresarial, sino también para ser replicados en otros estudios de caso. No obstante, se reconoce que la ausencia de otros indicadores, como el conocimiento de los estados financieros y la participación de un número mayor de empresas en las técnicas de muestreo, podría ser una limitación para concretar y generalizar los resultados. A pesar de ello, se considera que con la información aquí utilizada se marca una tendencia clara en cuanto a la relación actual entre los sistemas de calidad con el desempeño empresarial, además que abre la posibilidad a nuevos estudios que se basen en el método propuesto.

\section{AGRADECIMIENTOS}

Esta investigación ha sido financiada por el Consejo Nacional de Ciencia y Tecnología (CONACyT), bajo el proyecto de tesis doctoral Sistemas de Calidad en establecimientos de Tipo Inspección Federal. Del mismo modo, los autores agradecen la colaboración de las empresas estudiadas, que han facilitado los datos necesarios para realizar este trabajo.

\section{REFERENCIAS}

Ahmed, S., y Hassan, M. (2003). Survey and case investigations on application of quality management tools techniques in SMIs. International Journal of Quality \& Reliability Management, 20(7), 795-826. doi: 10.1108/02656710310491221

Álvarez-García, J., Vila-Alonso, M., Fraiz-Brea, J., y Río-Rama del, M.C. de la. (2014). Relación entre herramientas y factores críticos de la calidad. Revista Europea de Dirección y Economía de la Empresa, 23(2), 82-97. doi: 10.1016/j.redee.2012.11.004

Boyer, R., y Freyssenet, M. (2003). Los modelos productivos. Madrid, España: Editorial Fundamentos. 
Calantone, R.J., Cavusgil, S.T., y Zhao, Y. (2002). Learning orientation, firm inovation capability, and firm performance. Industrial Marketing Management, 31(6),515-524. doi: 10.1016/S0019-8501 (01)00203-6

Camisón, C., Boronat-Navarro, M., Villar-López, A., y Puig-Denia, A. (2009). Sistemas de gestión de la calidad y desempeño: Importancia de las prácticas de gestión del conocimiento y de I+D. Revista Europea de Dirección y Economía de la Empresa. 18(1), 123-134.

Chavez, J.H., y Torres-Rabello, R. (2012). Supply Chain Management. Santiago: RIL editores.

Chikuku, T., Chinguwa, S., y Macheka, M. (2012). Evaluation of the impact of obtaining ISO 9001:2008 Quality Management System (QMS) certifications by manufacturing companies in Zimbabwe. International Journal of Engineering Science and Technology, 4(8), 4168-4186.

Dale, B., y Mcquater, R. (1998). Managing business improvement and quality: Implementing key tools and techniques. Oxford, England: Blackwell Publishing.

Fideicomisos Instituidos en Relación a la Agricultura. (2014). Panorama del porcino. Recuperado de http://www.financierarural.gob.mx/ informacionsectorrural/Panoramas/Ficha\%20Porcino.pdf

Heras, I., Bernardo, M., y Casadesús, M. (2007). La integración de sistemas de gestión basados en estándares internacionales: resultados de un estudio empírico realizado en la CAPV. Revista de Dirección y Administración deEmpresas, (14), 155-174.

Heras, I., y Landín, G.A. (2011). Impacto de la certificación ISO 14001 en el rendimiento financiero empresarial: Conclusiones de un estudio empírico. Cuadernos de Economía y Dirección de la Empresa, 14(2), 112-122. doi:10.1016/j.cede.2011.02.002

Heras, I., Landín, G.A., y Casadesús, M. (2006). A Delphi Study on Motivation for ISO 9000 and EFQM. International Journal of Quality \& Reliability Management, 23(7), 807-827. doi: 10.1108/02656710610679824

Heras, I., Marimon, F., y Casadesús, M. (2009). Impacto competitivo de las herramientas para la gestión de la calidad. Cuadernos de 
Economía y Dirección de la Empresa, 12(41), 7-36. doi:10.1016/ s1138-5758(09)70046-5

International Organization for Standarization. (2005). Sistemas de gestión de la calidad: Fundamentos y vocabulario. Recuperado de https://www.iso.org/obp/ui/\#iso:std:iso:9000:ed-3:v1:es:term:3.2.5

Llopis, J., y Tarí-Guilló, J.J. (2003). The Importance of Internal Aspects in Quality Improvement. International Journal of Quality \& Reliability Management, 20(3), 304-324. doi: 10.1108/02656710310461314

Lo, C.K.Y., Yeung, A.C.L., y Edwin-Cheng, T.C. (2011). Meta-Standards, Financial Performance and Senior Executive Compensation in China: An Institutional Perspective. International Journal of Production Economics, 129(1), 119-126. doi:10.1016/j.ijpe. 2010.09.011

López-Palacios, M.G., Muñoz-Rodríguez, M., Leos-Rodríguez, J.A., y Cervantes-Escoto, F. (2010). Innovación en valor en la industria cárnica bovina mexicana: Estrategias que adoptan los líderes de Mercado. Revista Mexicana de Ciencias Pecuarias, 1(4), 417-432.

Mahnaz, F. (2014). Impact of ISO 9000 on Business Performance in Pakistan: Implications for Quality in Developing Countries. Quality Management Journal, 21(1), 16-24.

Marín-Vinuesa, L.M. (2013). Gestión de la Calidad Total e indicadores no financieros: Reflejo del valor de la certificación ISO 9001:2000. Revista Europea de Dirección y Economía de la Empresa, 22(2), 97-106. doi:10.1016/j.redee.2012.11.002

Martínez-Costa, M., y Martínez-Lorente, Á.R. (2008). Sistemas de gestión de calidad y resultados empresariales: Una justificación desde las teorías institucional y de recursos y capacidades. Cuadernos de Economía y Dirección de la Empresa, 11(34), 7-31. doi: 10.1016/S1138-5758(08)70051-3

Morelos-Gómez, J., Fontalvo, T.J., y Vergara, J.C. (2013). Incidencia de la certificación ISO 9001 en los indicadores de productividad y utilidad financiera de empresas de la zona industrial de Mamonal en Cartagena. Estudios Gerenciales, 29(126), 99-109. doi: 10.1016/S0123-5923(13)70025-1 
Moura-Duarte, A.L.C., Ledur-Brito, L.A., Di-Serio, L.C., y SilveiraMartins, G. (2011). Operational Practices and Financial Performance: An Empirical Analysis of Brazilian Manufacturing Companies. Brazilian Administration Review, 8(4), 395-411. doi: 10.1590/S1807-76922011000400004

Nava-Carballido, V.M., y Rivas-Tovar, L.A. (2008). Desempeño de las organizaciones mexicanas certificadas en la norma ISO 9001:2000. Estudios Gerenciales, 24(108), 107-128. doi: 10.1016/ S0123-5923(08)70046-9

Organización de las Naciones Unidas para la Alimentación y la Agricultura. (2004a). Las normas sociales y ambientales, la certificación y el etiquetado de cultivos comerciales. Recuperado de http://www.fao.org/docrep/008/y5136s/y5136s00.htm

Organización de las Naciones Unidas para la Alimentación y la Agricultura.(2004b). Calidady competitividad de la agroindustria rural de América Latina y el Caribe: Uso eficiente y sostenible de la energía. Recuperado de http://www.fao.org/documents/card/ es/c/20b4ae59-5140-55a5-a441-64faa86c4f5f/

Parra, C.M., Villa, V.M., y Restrepo, J.W. (2009). Gestión de la calidad con el modelo EFQM en 10 PyMEs metalmecánicas de Medellín. Revista EIA, (11), 9-19.

Pérez-Méndez, J.A., y Machado-Cabezas, Á. (2015). Relationship between Management Information Systems and Corporate Performance. Revista de Contabilidad, 18(1), 32-43. doi: 10.1016/ j.rcsar.2014.02.001

Romero, C. (1996). Análisis de las decisiones multicriterio. Madrid: Isdefe.

Secretaría de Agricultura, Ganadería, Desarrollo Rural, Pesca y Alimentación. (2014). México exportó 86 mil 294 toneladas de carne de cerdo en 2013. Recuperado de: http://www.sagarpa.gob. $\mathrm{mx} / \mathrm{saladeprensa/2012/Paginas/2014B401.aspx}$

Secretaría de Agricultura, Ganadería, Desarrollo Rural, Pesca y Alimentación. (2016). Directorio de establecimientos TIF. Recuperado de: http://www.senasica.gob.mx/?doc=23949

Sánchez-Ollero, J.L., García-Pozo, A., y Marchante-Mera, A.J. (2014). Análisis de la incidencia de las certificaciones de calidad sobre la 
productividad de los hoteles en Andalucía. Universia Business Review, (44), 88-103.

Shepherd, W.G. (1999). The Economics of Industrial Organization. USA: Waveland Press.

Tarí-Guilló, J.J., y Pereira-Moliner, J. (2012). Calidad y rentabilidad: Análisis del certificado Q en las cadenas hoteleras. Universia Business Review, (34), 52-67.

Ul- Hassan, M., Mukhtar, A., Ullah-Qureshi, S., y Sharif, S. (2012). Impact of TQM Practices on Firm's Performance of Pakistan's Manufacturing Organizations. International Journal of Academic Research in Business and Social Sciences, 2(10), 232-259.

US Department of Agriculture. (2015). Livestock and Poultry: World Markets and Trade. Recuperado de http://apps.fas.usda.gov/ psdonline/circulars/livestock_poultry.PDF 
